\title{
Moderate to severe Hallux Valgus Deformities. Surgical treatment with SCARF Osteotomy Technique.
}

\author{
Julian Ruci *, Eduard Gjika
}

Received: 15 November 2021 / Accepted: 07 December 2021 / Published online: 20 January 2022

This article is published with open access at https://journal.astes.org.al

(C) The author(s) 2022. \& Copyright (C) 2022, the Albanian Society for Trauma and Emergency Surgery

(c) The Albanian Journal of Trauma and Emergency Surgery is an Open Access Journal. All articles are distributed under the terms of the Creative Commons Attribution Non-Commercial License: http://creativecommons.org/licenses/by-nc/4.0/) which permits unrestricted non-commercial use, distribution, and reproduction in any medium provided the original work is properly cited.

\begin{abstract}
Introduction: Many procedures are described in the literature for the surgical management of hallux valgus. There are over 130 surgical procedures described. There is ever rising enthusiasm among orthopedic surgeons regarding diaphyseal osteotomy ever since Burutaran described the procedure in 1973.

We report our experience of treating severe cases of Hallux Valgus deformity with a SCARF osteotomy at the last three years followup, at patients diagnosed and operated in the University Hospital of Trauma, from January 2015 - February 2018. The technique we are presenting, provides the correction of moderate to severe hallux valgus deformities.

Material and Methods: Correction of hallux valgus deformities was achieved using a $\mathrm{Z}$ step osteotomy cut to realign the first metatarsal bone. A retrospective analysis was undertaken in 38 consecutive patients ( 54 feet). All results were analyzed by clinical examination, a questionnaire including the AOFAS forefoot score, modified, and plain roentgenograms.

Results: Hallux valgus and intermetatarsal angle improved at mean $19.6^{\circ}$ and $6.9^{\circ}$, respectively. Mean forefoot score improved from 50.1 to 91 points out of 100 possible points. Satisfactory healing time was expressed by an average return back to their attitude of 6-12 weeks, without including physiotherapy. Persistence or recurrence of hallux valgus was seen in 3 patients (8\%).

The complication rate was $5.4 \%$ including superficial wound infection, atrophy of the muscles, traumatic dislocation of the distal fragment.

Conclusion: Scarf osteotomy is a powerful and versatile procedure to correct hallux valgus deformity, and provides a predictable and satisfying result. Scarfs are not considered as a single osteotomy but as a combination of several procedures and displacement in several planes are possible.
\end{abstract}

Keywords: metatarsal bone, osteotomy, angle, restore deformity,

\section{Abbreviations}

American Orthopedic Foot and Ankle Society - AOFAS; Hallux Valgus - HV; First Metatarsophalangeal Joint MTPJ1; Intermetatarsal - IM;

Original article, no submission or publication in advance or in parallel

\section{* Corresponding author}

Julian Ruci MD

$\triangle$ julian.ruci@yahoo.com

University Hospital of Trauma, Tirana, ALBANIA

\section{Introduction}

Numerous osteotomies have been described for surgical treatment of the primary varus metatarsus component of the hallux valgus deformity. Over 130 metatarsal osteotomy techniques have been described in the world literature for surgical treatment of hallux valgus.

In clinical practice, most orthopedists limit these to a few procedures that invariably involve soft tissue release, medial exostostomy combined with proximal or distal osteotomy of the first metatarsal.

The result of surgical correction is sometimes unsatisfactory for the orthopedist and undesirable for the patient.

Any surgical procedure should result in a congruent metatarsal-phalangeal joint first, painless movement, improved makeup, and the ability to wear the desired shoes. 
The procedure should also result in as few complications as possible and should be easily feasible. The first osteotomy of the metatarsal scarf has significantly improved correction in hallux valgus and has recently gained popularity among European surgeons.[4]

It can extend to a wide range of indications and result in early functional recovery with a greater number of minor complications.

The angle between the first and second metatarsals is between 8 to 9 degrees, usually considered to be the upper limits of normal. The valgus angle of the first metatarsophalangeal joint also is more than 15 to 20 degrees considered to be the upper limits of normal. If the valgus angle of the first metatarsophalangeal joint exceeds 30 to 35 degrees, pronation of the great toe usually results.

This abnormal rotation, leads the abductor hallucis, which is normally plantar to the flexion-extension axis of the first metatarsophalangeal joint, to move further plantar ward. The only restraining medial structure is the medial capsular ligament with its capsule-sesamoid portion (inserting into the base of the proximal phalanx) -and capsule-phalangeal portion (inserting into the plantar plate). The adductor hallucis, which is unopposed by the abductor hallucis, pulls the great toe further into valgus, stretching the medial capsular ligament and allowing the metatarsal head to drift medially from the sesamoids. (fig.1,2)

In addition, the flexor hallucis brevis, flexor hallucis longus, adductor hallucis, and extensor hallucis longus increase the valgus moment at the metatarsophalangeal joint, further deforming the first ray. The sesamoid ridge on the plantar surface of the first metatarsal head flattens because of pressure from the tibial sesamoid.

With this point of view, the normal or increased distal metatarsal articulation angle, bone stock, and symptomatic
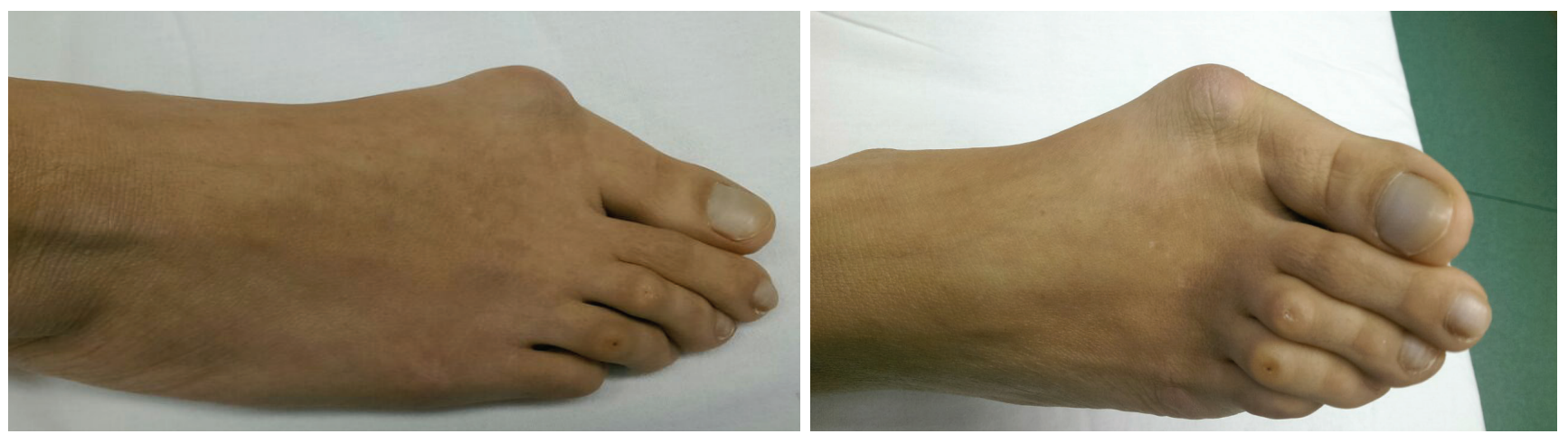

Figure 1. Hallux Valgus, severe bunion
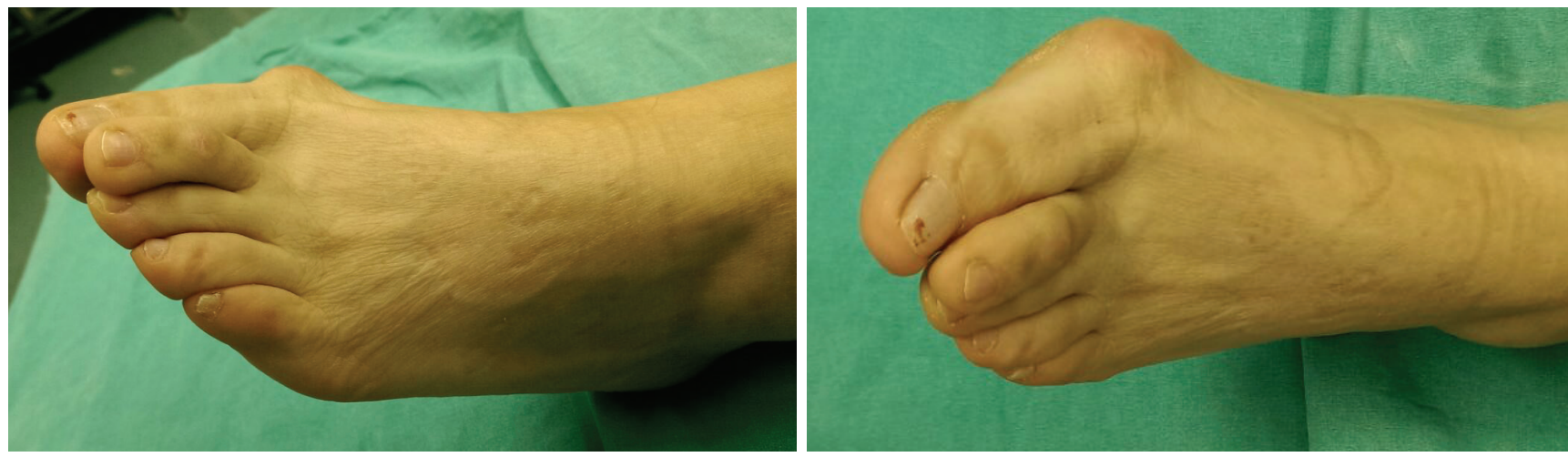

Figure 2. Hallux Valgus, extreme bunion
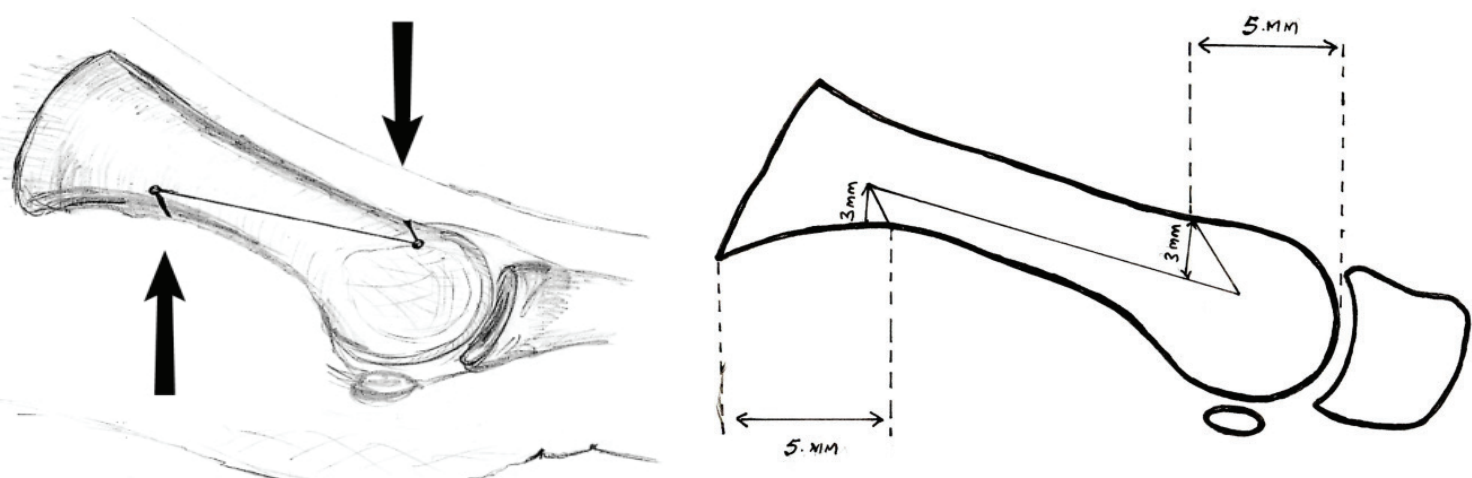

Figure 3. Z osteotomy cuts and measurements 
hallux valgus (HV) deformity have been established as major indications for the SCARF osteotomy.

The osteotomy for the correction of the first metatarsal bone to reduce an increased intermetatarsal angle was performed using a $Z$ step cut. SCARF is the term used for this $\mathrm{Z}$ step osteotomy and osteosyntheses technique [1, 2]. Since the first description of SCARF Osteotomy, this procedure has been used with great success for correction of moderate to severe hallux valgus deformities. [12] (fig.3)

The principle of this osteotomy technique in search for greater stability of the corrective first metatarsal osteotomies. Micro oscillating saws allowed angulated osteotomy cuts in bone. Early weight bearing due to great stability and rare postoperative complications have contributed to its frequent application. Sagittal-plane instability frequently leads to prolonged osseous healing and first metatarsal dorsiflexion malposition [2]. Therefore, midshaft osteotomies may fill the gap between the limitation of distal osteotomies and the instability of proximal osteotomies

To date only a few reports exist in the literature describing midterm results of SCARF osteotomy in larger populations. The literature concerning the SCARF osteotomy includes technical notes, but indications and contraindications have not been well defined. The current authors report their experience with a modified SCARF procedure in a three-year follow-up and indicate the use of this procedure in compare to other corrective procedures.

\section{Clinical and Radiological Assessment}

Clinical preoperative and follow-up evaluation was obtained by using the 100-point AOFAS forefoot score, modified. Follow-up examination and rating was done by the same team involved in performing the surgical procedure. Most of patients seen in clinic were photographed and overall radiographic assessment was done on weight-bearing $\mathrm{x}$-rays obtained pre- and postoperatively in standardized dorsoplantar and lateral views. (fig. 4)

\section{Surgical Technique}

Operations were performed using spinal locoregional anesthesia or nerve block anesthesia Also a tourniquet just above the knee joint was used. The standard approach was via a medial incision, at the junction of the plantar and the dorsal skin, with its proximal part below the surface projection of the metatarsi. The joint capsule and the medial collateral ligament of the MTPJ1 were incised horizontally, at the line of the first incision. The medial aspect of the metatarsal head was exposed. The medial eminence of the metatarsal head was partially resected. From an additional small dorsal interdigital approach, the lateral capsule was released longitudinally above the lateral sesamoid, leaving the plantar plate and the adductor tendon intact. The sesamoids were then mobilized trying to turn them back to the anatomic position. (fig.5)

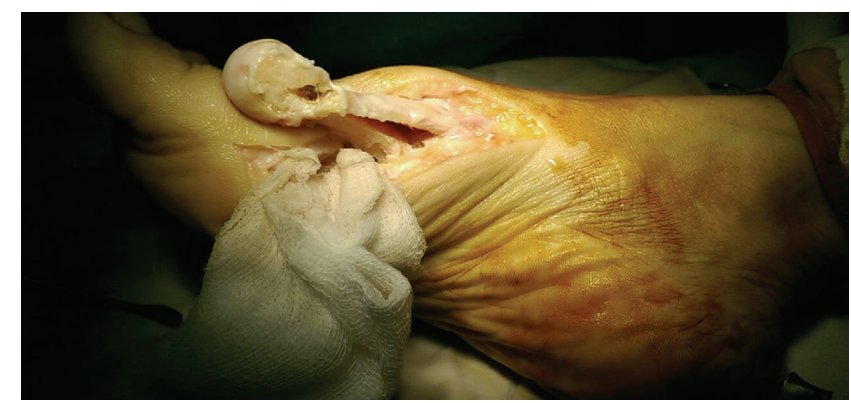

Figure 5. Technique SCARF exposition of MTPJ1;

In preparation for the three osteotomy cuts, two guiding 1.2-mm K wires were inserted at the corner points of the planned SCARF cut as entry point of the proximal pin averaged $1 \mathrm{~cm}$ distal to the first metatarsal medial cuneiform joint line, over the concavity of the inferior aspect of the metatarsal at the junction of the plantar inferior to the medial aspect.

The entrance point of the distal pin crossed the metatarsal head $5 \mathrm{~mm}$ proximal to the dorsal cartilage surface in the dorsal to the medial aspect.
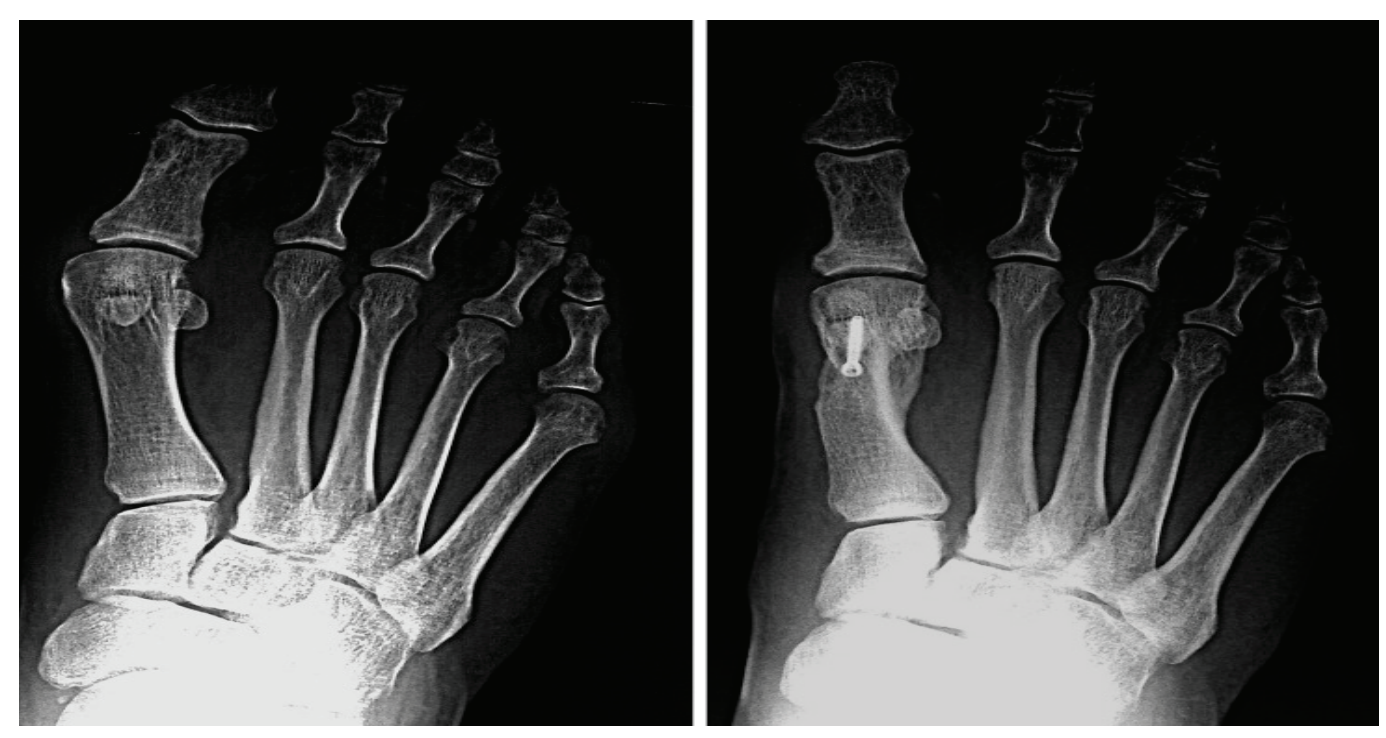

Figure 4. Pre- and post-operative $X$-ray 
Both $\mathrm{K}$ wires were oriented parallel to each other. The horizontal osteotomies were performed using an oscillating saw.

The angle of each cut was at $45^{\circ}$ to $60^{\circ}$ to the longitudinal metatarsal axis.

The authors' modification of the SCARF osteotomy described by Barouk includes the angulation of the distal osteotomy cut and the placement of the distal pin on the dorsal-medial aspect of the first metatarsal $[2,3]$.

After completing the osteotomy, the distal fragment was displaced laterally to reduce the intermetatarsal angle. Lateral displacement was achieved by pushing the distal fragment laterally while holding the proximal fragment of the first metatarsal in place. The translation is greater for larger IM angles. For maximal stability of the osteotomy after translation, it is necessary to orient the proximal and distal osteotomy cut strictly parallel to each other. Translation and lowering were indicated for hallux valgus with intermittent metatarsalgia or a deficit of the first metatarsal head in weight-bearing. This is best diagnosed clinically. (fig. 6)

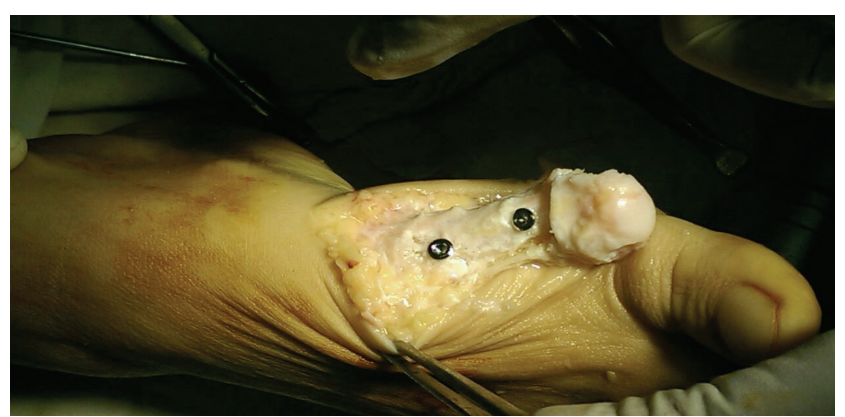

Figure 6. Technique SCAR, osteosynthesis.

The osteotomy was modified by directing the orientation of the $\mathrm{K}$ wires. Translation and shortening of the first metatarsal could be performed. Shortening was obtained by increasing the obliquity of the anterior and posterior cuts to the longitudinal axis of the second metatarsal. Additional shortening was indicated in severe forefoot deformity with luxation of the lesser toes at the metatarsal-phalangeal joints. Translation and lengthening were indicated in cases with short first metatarsal combined

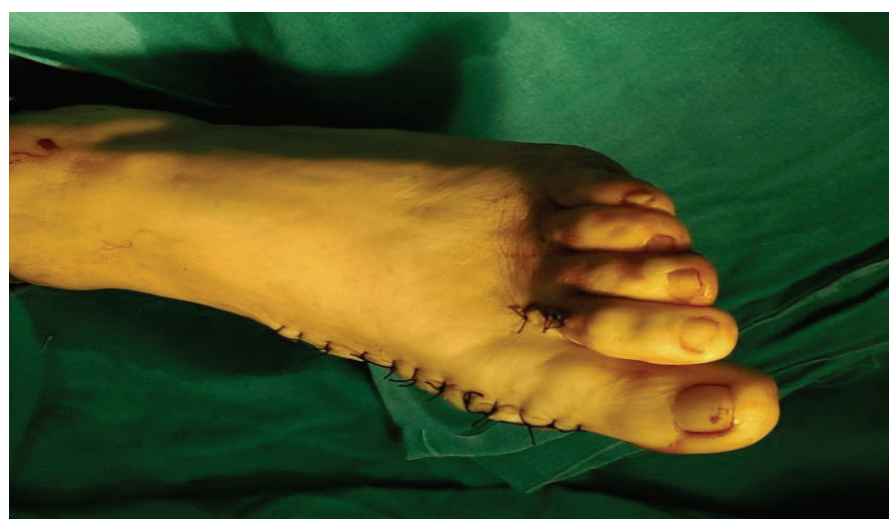

Figure 7. Technique SCAR, postoperative status. with metatarsalgia. Fixation of the osteotomy was achieved using a small cannulated bi-cortical compressive screw of steel or titanium material. (fig. 6,7)

\section{Results}

In our study were $30(78.9 \%)$ female and $8(21.1 \%)$ male patients. The average age at the time of surgery was 50 years. the range, 35 to 65 years. $2(5.2 \%)$ of the patients had systemic disorders which caused neuropathy and/or angiopathy of the lower extremity.

There were $2(5.2 \%)$ of the patients with diabetes mellitus, $5(13.1 \%)$ of the patients with rheumatologic symptoms. Patients were informed about a higher risk for possible wound healing complications.

If they still wanted the operative treatment, surgery was planned. Patients were also informed to stop smoking, but none did. $5(13.1 \%)$ of the patients had previous hallux valgus operations of different types. Akin osteotomy was performed in $7(18.4 \%)$ of cases. Akin osteotomy normally is added to SCARF osteotomy in those cases where hallux valgus inter phalanges angle is greater than $15^{\circ}$, or after completing the Scarf osteotomy and the soft-tissue reconstruction, the hallux was still in more than $10^{\circ}$ of valgus position, as studied in the literature.

In $6(16.7 \%)$ of cases for treatment of metatarsalgia, was performed nerve removal. $20(52.6 \%)$ of the patients underwent surgery on both feet.

All of them in two sessions with an average interval of six months. At mean of 12 to 24 months a retrospective analysis including clinical examination and radiographic evaluation was undertaken.

Regarding to postoperative pain, 27 (71\%) of operated feet reported to be completely pain-free, and 5(13.1\%) of operated feet reported to have only occasional or slight discomfort.

The remaining $6(16.7 \%)$ of cases had mild to moderate discomfort.

The length of the first metatarsal was reduced by an average of $2.5 \mathrm{~mm}(+/-2.7 \mathrm{~mm})$. Healing was expressed by the ability of full weight bearing gait pattern and the time from surgery to return back to work. Bone healing was

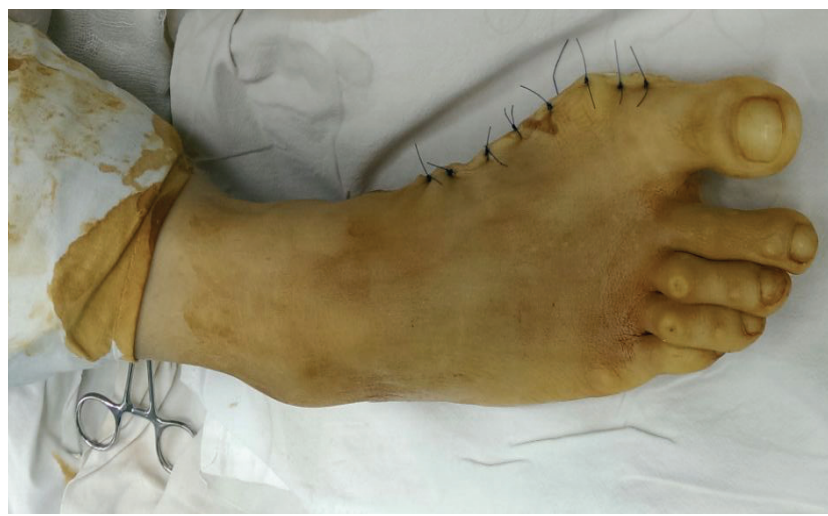


documented on plain radiographs six weeks after surgery.

At the time of final follow-up, no loss of correction of the intermetatarsal angle was noted.

However, $4(10.5 \%)$ patients showed a recurrent or persistent hallux valgus.

Complications were rare and comprised superficial wound infections necessitating antibiotic medication and traumatic dislocation of the osteosyntheses.

\section{Discussion}

More than 130 procedures are described for the surgical treatment of hallux valgus. These include distal osteotomies (Chevron, Mitchell, Wilson osteotomies) or proximal metatarsal osteotomies. [4, 9]

The procedure is already known by orthopedists especially in Europe is the $Z$ diaphyseal step incision osteotomy called as scarf osteotomy. The scarf is the English translation of the phrase "le trait de Jupiter des Charpentiers" - a technique used by carpenters to increase the size of an entrance by cutting two corresponding beams. $[4,10,11]$

In 1973 Burutaran described a similar incision in the first metatarsal for the extension of the first ray. [4]

Zygmunt in 1983 began performing $\mathrm{Z}$ incisions with lateral displacement of the first metatarsal head. [10]

The technique was further developed by Borrelli and Weil in 1984, they gave the name "scarf" to this technique, [4] in Europe Barouk has done more than 2300 scarf osteotomies.

Scarring is a combined procedure which involves resection of the median eminence and medial capsular fold, metatarsal osteotomy, lateral soft tissue release, and if required proximal phalanx osteotomy (Akin). [1] Procedures are performed in combination.

The SCARF osteotomy has become a widely used procedure in middle Europe since the introduction and the development of specially designed osteosyntheses material.

In our study were $30(78.9 \%)$ female and $8(21.1 \%)$ male patients. The average age at the time of surgery was 50 years. the range, 35 to 65 years.

Nix et al. [13] pooled prevalence estimates for $\mathrm{HV}$ were $23 \%$ in adults aged $18-65$ years and $35.7 \%$ in elderly people aged over 65 years, and prevalence increased with age and was higher in females (30\%) compared to males (13\%) have comparable results to those of our study.

Akin osteotomy was performed in 7(18.4\%) of cases. Akin osteotomy normally is added to SCARF osteotomy in those cases where hallux valgus inter phalanges angle is greater than $15^{\circ}$, or after completing the Scarf osteotomy and the soft-tissue reconstruction, the hallux was still in more than $10^{\circ}$ of valgus position, as studied in the literature.

In $6(16.7 \%)$ of cases for treatment of metatarsalgia, was performed nerve removal. $20(52.6 \%)$ of the patients underwent surgery on both feet.
All of them in two sessions with an average interval of six months. At mean of 12 to 24 months a retrospective analysis including clinical examination and radiographic evaluation was undertaken.

Regarding to postoperative pain, 27 (71\%) of operated feet reported to be completely pain-free, and $5(13.1 \%)$ of operated feet reported to have only occasional or slight discomfort.

The remaining $6(16.7 \%)$ of cases had mild to moderate discomfort.

The length of the first metatarsal was reduced by an average of $2.5 \mathrm{~mm}(+/-2.7 \mathrm{~mm})$. Healing was expressed by the ability of full weight bearing gait pattern and the time from surgery to return back to work. Bone healing was documented on plain radiographs six weeks after surgery.

At the time of final follow-up, no loss of correction of the intermetatarsal angle was noted.

However, $4(10.5 \%)$ patients showed a recurrent or persistent hallux valgus.

This paper, introducing the SCARF osteotomy, describes the operative procedure and the possibilities of the SCARF in combination with other osteotomies in lesser metatarsals.

Complications included two metatarsal fractures, failure to consolidation, also, neuropathy and angiopathy.

Conclusion: Scarf osteotomy is a powerful and versatile procedure to correct hallux valgus deformity, and provides a predictable and satisfying result. Scarfs are not considered as a single osteotomy but as a combination of several procedures and displacement in several planes are possible

\section{Declaration of Conflicting Interests and Ethics;}

The authors declare no conflict of interest. This research study complies with research publishing ethics. The scientific and legal responsibility for manuscripts published in Albanian Journal of Trauma and Emergency Surgery - AJTES, belongs to the author(s).

\section{Acknowledgements: None.}

\section{References}

1. Akin, OF: The treatment of hallux valgus: a new operative procedure and its results. Med Sentinel 33:678-679, 1925

2. K.H. Kristen, C. Berger, S. Stelzig, E. Thalhammer, M. Posch, A. Engel The Scarf osteotomy for Hallux Valgus Vienna, Austria FOOT \& ANKLE INTERNATIONAL Copyright C 2002 by the American Orthopaedic Foot \& Ankle Society, Inc

3. Barouk, LS: The first metatarsal Scarf osteotomy associated with the first phalanx osteotomy in the hallux valgus treatment. Extrait de medicine et chirurgie du pied. EFORT, Foot and ankle speciality day: 133-160, 1993

4. Barouk, LS: Scarf osteotomy of the first metatarsal in the treatment of hallux valgus. Foot Diseases 2(1):35-48, 1995 
5. Besse, JL; Langlois, F; Berthonnaud, E; et al: Semi automated $\mathrm{X}$-ray assessment of 50 hallux valgus cases treated by Scarf osteotomy, using the Piedlog software. Synopsis book, Second internat. AFCP spring meeting, Bordeaux May, 2000

6. Bonnel, F; Canovas, F; Poiree, G; et al: Evaluation of the Scarf osteotomy in hallux valgus related to distal metatarsal articular angle: a prospective study of 79 operated cases. Rev Chir Orthop Reparatrice Appar Mot. July 85 (4):381-6, 1999

7. Crosby, LA; Bozarth, GR: Fixation comparison for Chevron osteotomies. Foot \& Ankle Int'1, 19(1):41-43, 1998

8. Kitaoka, HB; Alexander, IJ; et al: Clinical rating systems for the ankle-hindfoot, midfoot, hallux, and lesser toes. Foot \& Ankle Int'l 15(7):349-53, 1994

9. Robinson AH, Limbers JP. Modern concepts in the treatment of hallux valgus. J Bone Joint Surg Br 2005. Aug;87(8):10381045 10.1302/0301-620X.87B8.16467 [PubMed]

[CrossRef] [Google Scholar]
10. Weil LS. Scarf osteotomy for correction of hallux valgus. Historical prospective, surgical technique and results. Foot Ankle Int 2000;5:559-580 [PubMed] [Google Scholar]

11. Jones S, Al Hussainy HA, Ali F, Betts RP, Flowers MJ. Scarf osteotomy for hallux valgus. A prospective clinical and pedobarographic study. J Bone Joint Surg Br 2004. Aug;86(6):830-836 10.1302/0301-620X.86B6.15000 [PubMed] [CrossRef] [Google Scholar]

12. S.S.Suresh. Scarf osteotomy - Is it the procedure of choice in hallux valgus surgery? A preliminary report. Oman Med $\mathrm{J}$. 2007;22(3):47-50.

13. Nix S, Smith M, Vicenzino B. Prevalence of hallux valgus in the general population: a systematic review and metaanalysis. J Foot Ankle Res. 2010; 3:21. Published 2010 Sep 27. doi:10.1186/1757-1146-3-21 